\title{
Weighted Bergman kernel: asymptotic behavior, applications and comparison results
}

\author{
by \\ Bo-Yong Chen (Shanghai)
}

\begin{abstract}
Inspired by the work of Engliš, we study the asymptotic behavior of the weighted Bergman kernel together with an application to the Lu Qi-Keng conjecture. Some comparison results between the weighted and the classical Bergman kernel are also obtained.
\end{abstract}

1. Introduction. Let $D$ be a domain in $\mathbb{C}^{n}$, and $\varrho$ a measurable nonnegative function on $D$. Let $L^{2}(D, \varrho)$ denote all square-integrable functions associated to the measure $\varrho d V$, where $d V$ is the Lebesgue measure. Furthermore, $H^{2}(D, \varrho):=L^{2}(D, \varrho) \cap \mathcal{O}(D)$. Following Z. Pasternak-Winiarski [25], the weight function $\varrho$ is called admissible if $H^{2}(D, \varrho)$ is a closed subspace of $L^{2}(D, \varrho)$ and for any $z \in D$ the evaluation functional $\tau_{z}: H^{2}(D, \varrho) \rightarrow \mathbb{C}$ defined by $\tau_{z}(f)=f(z)$ is a bounded linear functional on $H^{2}(D, \varrho)$. According to the Riesz representation theorem there is a reproducing kernel $K_{\varrho}(z, w):=K_{D, \varrho}(z, w)$, which is called the Bergman kernel with weight $\varrho$. When $\varrho \equiv 1$, it is just the (classical) Bergman kernel $K_{D}$. A sufficient condition for a weight to be an admissible weight was given in [25]: if the function $\varrho^{-a}$ is locally integrable with respect to the Lebesgue measure for some $a>0$, then $\varrho$ is an admissible weight. In particular, $\varrho$ is admissible provided that $1 / \varrho \in L_{\text {loc }}^{\infty}(D)$.

In [11], Engliš proved that if $\phi, \psi>0,1 / \psi \in L_{\mathrm{loc}}^{\infty}(D),-\log \phi$ is a convex function and $1 \in H^{2}\left(D, \phi^{k} \psi\right)$ for some $k$, then

$$
\lim _{k \rightarrow \infty} K_{\phi^{k} \psi}(z, z)^{1 / k}=1 / \phi(z) .
$$

This asymptotic formula plays an important role in the theory of Berezin quantization: one always takes $-\log \phi$ to be the potential of some Kähler

2000 Mathematics Subject Classification: Primary 32A25.

Key words and phrases: weighted Bergman kernel, Lu Qi-Keng conjecture.

Supported by The Rising Star Project for Young Researchers of Shanghai No. 05QMX1452 and a grant from Chinese Excellent Doctorate's Degree Theses; partially supported by NSFC No. 10571135. 
metric $d s^{2}=\sum_{j, k} g_{j k} d z_{j} d \bar{z}_{k}$ and $\psi=\operatorname{det}\left(g_{j \bar{k}}\right)$ (cf. [11] for details). It was naturally asked by Engliš whether (1) still holds if one replaces the hypothesis of convexity by plurisubharmonicity. We will first give a partial answer to this question.

Given domain $D$ in $\mathbb{C}^{n}$, we denote by $\operatorname{PSH}(D)$ the set of all psh functions on $D$. Following Klimek [18], the pluricomplex Green function with a logarithmic pole at $w \in D$ is defined as follows:

$$
g_{D}(z, w):=\sup \{u(z)\}
$$

where the supremum is taken over all negative functions in $\operatorname{PSH}(D)$ satisfying $u(z) \leq \log |z-w|+O(1)$ near $w$.

Theorem 1.1. Let $D$ be a pseudoconvex domain in $\mathbb{C}^{n}$ such that

$$
g_{D}(z, w) \sim \log |z-w|
$$

near $w$ for any $w \in D$. Let $\phi, \psi>0$ be so that $-\log \phi$ is psh and $1 / \psi \in$ $L_{\mathrm{loc}}^{\infty}(D)$. Suppose that there is a number $k_{0}>0$ such that the function $-k_{0} \log \phi-\log \psi$ is bounded from below by a psh function $\lambda \in L_{\mathrm{loc}}^{\infty}(D)$. Then (1) holds.

The hypothesis on $D$ holds for all bounded domains, hyperconvex domains, domains carrying a bounded strictly psh function in $\mathbb{C}^{n}$, and hyperbolic domains in the plane (see Section 2). We verify (1) for bounded pseudoconvex domains with the Kähler-Einstein metric or the Bergman metric.

Engliš [11] also found a simple but interesting fact: if the asymptotic equality (1) holds and $\phi$ satisfies a certain condition $(1 / \phi$ cannot be extended to a sesqui-holomorphic function on $D \times \bar{D})$, then $K_{\phi^{k} \psi}(z, w)$ has a zero for all sufficiently large $k$. On the other hand, Ligocka [20] showed that the Bergman kernel of a certain Hartogs domain can be expressed as the sum of a series of weighted Bergman kernels defined on another domain of lower dimension. These facts enable one to find zeros of the Bergman kernel for various Hartogs domains. We set

$$
D_{m, \varphi}:=\left\{(z, t) \in D \times \mathbb{C}^{m}:|t|^{2}<\varphi(z)\right\}
$$

where $D$ is a domain in $\mathbb{C}^{n}$ and $\varphi$ is a positive function on $D$. It is well known that $D_{m, \varphi}$ is pseudoconvex iff $D$ is pseudoconvex and $-\log \varphi$ is psh.

Theorem 1.2. Let $D$ be as in Theorem 1.1.

(i) Suppose that $\phi>0,-\log \phi \in \operatorname{PSH}(D)$ and $1 / \phi \in L_{\text {loc }}^{\infty}(D)$ fails to have a sesqui-holomorphic extension near a point $z^{0} \in D$. Then for any neighborhood $U$ of $z^{0}, K_{D_{m, \phi}}((z, 0),(w, 0))$ has a zero in $U \times U$ for all sufficiently large $m$.

(ii) Suppose that $\left\{\phi_{k}\right\}_{k=1}^{\infty}$ is a sequence of positive functions on D such that $-\log \phi_{k}$ are psh, $1 / \phi_{k} \in L_{\mathrm{loc}}^{\infty}(D)$ and $\phi_{k}^{1 / k}$ converge pointwise to a 
positive lower semicontinuous function $\phi$ so that $1 / \phi^{m}$ fails to have a sesquiholomorphic extension near a point $z^{0} \in D$ for some $m$. Then for any neighborhood $U$ of $z^{0}, K_{D_{m, \phi_{k}}}((z, 0),(w, 0))$ has a zero in $U \times U$ for all sufficiently large $k$.

A result somewhat weaker than (i) has been obtained by Engliš [12] under the assumption that $-\log \phi$ is convex. Finding zeros of the Bergman kernel relates to an old conjecture raised by Lu Qi-Keng [21]: if $D$ is a domain, then $K_{D}(z, w) \neq 0$ for all $z, w \in D$. A domain with this property is called a $L u Q i$ Keng domain. There are a lot of counterexamples to this conjecture (cf. Boas [4] for a survey). Theorem 1.2 provides an easy way to find such examples. Moreover, we can find, for any $k \geq 1$, a convex domain in $\mathbb{C}^{n(k)}$ with a $C^{k}$ boundary, where $n(k)$ depends on $k$, such that the zero set of the Bergman kernel accumulates at some point $\left(z^{0}, z^{0}\right)$, where $z^{0} \in \partial D$ (cf. Theorem 5.3). This gives a partial answer to a question posed by Diederich-Herbort [7]: is there a bounded convex domain with smooth boundary satisfying the above property? In that paper, they found a convex domain with $C^{1}$ boundary which has the above property. Such domains would provide negative answers to the question of whether the Bergman kernel, viewed as a mapping from $\overline{D \times D}$ to the Riemann sphere $\overline{\mathbb{C}}$, is continuous. It should be mentioned that the answer is affirmative for bounded strongly pseudoconvex domains (cf. [13]).

Another application of the weighted Bergman kernel is to give a sufficient condition for a domain to be Hilbert-Stein (see Theorem 6.1)

A formula of Ligocka gives a relationship between the classical and weighted Bergman kernel on domains of different dimensions. However, little is known about the equal dimensional case. It was shown in [9] that for any $s>-1$ there is a constant $C_{s}>1$ such that

$$
C_{s}^{-1} \delta_{D}(z)^{-s} \leq \frac{K_{s}(z, z)}{K_{D}(z, z)} \leq C_{s} \delta_{D}(z)^{-s}
$$

provided that $D$ is a bounded, smooth, strongly pseudoconvex domain. Here $\delta_{D}(z)$ is the euclidean boundary distance of $z$ and $K_{s}(z, z)=K_{\delta_{D}^{s}}(z, z)$. We will give a generalization of this result. A domain is called locally convexifiable if each $p \in \partial D$ has a neighborhood $U$ such that $D \cap U$ is biholomorphically equivalent to a bounded convex domain.

TheOREM 1.3. If $D$ is a bounded locally convexifiable domain in $\mathbb{C}^{n}$, then

$$
C_{s}^{-1} \delta_{D}(z)^{-s} \leq \frac{K_{s}(z, z)}{K_{D}(z, z)} \leq C_{s} \delta_{D}(z)^{-s}, \quad s>-1, z \in D .
$$

We also have a slightly weaker version of Theorem 1.3 for a very general class of pseudoconvex domains. 
Theorem 1.4. Let $D$ be a bounded pseudoconvex domain in $\mathbb{C}^{n}$. Assume that there exists a negative psh exhaustion function @ such that for some number $0<\alpha \leq 1$ and a constant $c>1$ the inequality

$$
c^{-1} \delta_{D}(z)^{\alpha} \leq-\varrho(z) \leq c \delta_{D}(z)^{\alpha}
$$

holds on D. Let $0<t<1$. Then

$$
C_{s, t}^{-1} \delta_{D}(z)^{-s(1-t)} \leq \frac{K_{s}(z, z)}{K_{D}(z, z)} \leq C_{s, t} \delta_{D}(z)^{-s(1-t)}, \quad s>-1, z \in D .
$$

Remark. 1. As already shown by Diederich-Fornæss [6], if $D$ is a $C^{2}$ bounded pseudoconvex domain, then there is a number $0<\alpha \leq 1$ such that $D$ has a defining function $\varrho_{1}$ with $-\left(-\varrho_{1}\right)^{\alpha}$ being psh. If $D$ is a smooth $B$-regular domain in the sense of Sibony [28], then the exponent $\alpha$ can be any number in $(0,1)$ and $(2)$ of Theorem 1.4 holds for any $-1<s<0$.

2. When $D$ is strongly pseudoconvex and $-\log \phi$ is psh, Diederich et al. [9] proved that $K_{\phi}(z, z) / K_{D}(z, z) \geq C \phi(z)^{-1}$. It remains open whether the inequality holds for general pseudoconvex domains.

2. Preliminaries. We first collect some properties of the weighted Bergman kernel:

1) Maximal property [27]:

$$
K_{\varrho}(z, z)=\sup \left\{|f(z)|^{2}: f \in H^{2}(D, \varrho), \int_{D}|f|^{2} \varrho d V \leq 1\right\} .
$$

This implies $K_{\varrho}(z, z)$ is decreasing with respect to the weight.

2) Translation formula [11]: if $g: D \rightarrow D^{\prime}$ is a biholomorphic mapping, then

$$
K_{\varrho}(z, z)=J_{g}(z) K_{\varrho \circ g^{-1}}(g(z), g(w)) \overline{J_{g}(w)}
$$

where $J_{g}$ denotes the Jacobian determinant of $g$.

3) Localization property: if $D$ is a bounded pseudoconvex domain, $\varrho$ is a positive function so that $-\log \varrho$ is psh on $D$ and $V \subset \subset U$ are two open neighborhoods of $p_{0} \in \partial D$, then

$$
K_{D, \varrho}(z, z) \geq C^{-1} K_{D \cap U, \varrho}(z, z)
$$

for all $z \in D \cap V$ (the proof is the same as in Ohsawa [24], although he only considered the classical Bergman kernel function).

4) Ligocka's formula [20]: Let $D$ be a domain in $\mathbb{C}^{n}$ and let $\varphi$ be a positive measurable function on $D$ such that $1 / \varphi \in L_{\text {loc }}^{\infty}(D)$. Define the Hartogs domain as

$$
D_{m, \varphi}:=\left\{(z, t) \in D \times \mathbb{C}^{m}:|t|^{2}<\varphi(z)\right\} .
$$


Then

$$
K_{D_{m, \varphi}}((z, t),(w, s))=\sum_{j=0}^{\infty} \frac{(j+m) !}{j ! \pi^{m}} K_{D, \varphi^{m+j}}(z, w)\langle t, s\rangle^{j}
$$

where $\langle$,$\rangle denotes the scalar product in \mathbb{C}^{n}$.

To verify the hypothesis that $g_{D}(z, w) \sim \log |z-w|$ near $w$ for any $w \in D$, it suffices to find a bounded psh function which has this property. Let us see some examples.

(a) If $D$ is a bounded domain in $\mathbb{C}^{n}$, then $g_{D}(z, w) \geq \log |z-w|-\log R_{D}$, where $R_{D}$ is the diameter of $D$. On the other hand, $g_{D}(z, w) \leq \log |z-w|+$ $O(1)$ near $w$. Hence the hypothesis is satisfied.

(b) If $D$ is a hyperbolic planar domain (that is, $D$ carries a bounded nonconstant subharmonic function or equivalently, $D$ carries a Green function), then the pluricomplex Green function is the classical (negative) Green function, hence $g_{D}(z, w)-\log |z-w|$ is harmonic near $w$.

(c) Let $D$ be a pseudoconvex domain carrying a bounded $C^{2}$ strictly psh function $u$. For arbitrary $w \in D$, we take a function $\chi$ which is compactly supported in $D$ and identically equal to 1 in a neighborhood of $w$. One can easily find a constant $a_{w}$ such that $\psi(z)=\chi(z) \log |z-w|+a_{w} u(z)$ is a psh function on $D$ with a logarithmic pole at $w$ which is bounded above by a constant depending only on $w$. Hence the hypothesis is satisfied.

3. An extension theorem of Ohsawa and applications. Recently, Ohsawa [24] obtained a general $L^{2}$-extension theorem from which one can deduce all the earlier extension theorems. However, to state this theorem completely is quite laborious. Here we present a special case which is sufficient to prove our theorems. Let $D$ be a domain in $\mathbb{C}^{n}$ and let $D^{\prime}$ be the intersection of $D$ and a complex linear subspace of dimension $k$. We denote by $\#\left(D^{\prime}\right)$ the space of all $\Psi \in \operatorname{PSH}(D)$ satisfying the following conditions:

(i) $D^{\prime} \subset \Psi^{-1}(-\infty)$;

(ii) for any $p \in D^{\prime}$, there is a neighborhood $U$ of $p$ in $D$ such that

$$
\sup _{U \backslash D^{\prime}}\left|\Psi(z)-2(n-k) \log d\left(z, D^{\prime}\right)\right|<\infty
$$

where $d\left(z, D^{\prime}\right)$ denotes the Euclidean distance from $z$ to $D^{\prime}$.

For each $\Psi \in \#\left(D^{\prime}\right)$, one can define a positive measure $d V[\Psi]$ on $D^{\prime}$ as the minimum element of the partially ordered set of positive measures $d \mu$ satisfying

$$
\int_{D^{\prime}} f d \mu \geq \limsup _{t \rightarrow \infty} \frac{2(n-k)}{\sigma_{2 n-2 k-1}} \int_{D} f e^{-\Psi} \chi_{R(\Psi, t)} d V
$$

for any nonnegative continuous function $f$ which is compactly supported 
in $D$. Here $\sigma_{m}$ denotes the volume of the unit sphere in $\mathbb{R}^{m+1}$, and $\chi_{R(\Psi, t)}$ denotes the characteristic function of the set

$$
R(\Psi, t)=\{x \in D:-t-1<\Psi<-t\} .
$$

Proposition 3.1 (see [24]). Let $D$ be a pseudoconvex domain in $\mathbb{C}^{n}$ and let $D^{\prime}$ be as above. Let $\Psi \in \#\left(D^{\prime}\right)$. Then for any holomorphic function $f$ on $D^{\prime}$ satisfying $\int_{D^{\prime}}|f|^{2} d V[\Psi]<\infty$, there exists a holomorphic function $F$ on $D$ such that $\left.F\right|_{D^{\prime}}=f$ and

$$
\int_{D}|F|^{2} e^{-\Psi} d V \leq 2^{8} \pi \int_{D^{\prime}}|f|^{2} d V[\Psi] .
$$

We define

$$
\begin{aligned}
\log c_{D}^{1}(z) & :=\liminf _{\zeta \rightarrow z} 2 n\left(g_{D}(\zeta, z)-\log |\zeta-z|\right), \\
\log c_{D}^{2}(z) & :=\limsup _{\zeta \rightarrow z} 2 n\left(g_{D}(\zeta, z)-\log |\zeta-z|\right), \\
c_{D}(z) & :=\frac{c_{D}^{1}(z)}{1+\log \left(c_{D}^{2}(z) / c_{D}^{1}(z)\right)} .
\end{aligned}
$$

If $g_{D}(\zeta, z) \sim \log |\zeta-z|$ near $z$, then $c_{D}(z)>0$.

TheOREM 3.2. Let $D$ be a pseudoconvex domain as in Theorem 1.1. Suppose that $1 / \phi \in L_{\mathrm{loc}}^{\infty}(D)$ and $-\log \phi \in \operatorname{PSH}(D)$. Then

$$
K_{\phi}(z, z) \geq 2^{-8} \pi^{-1} \frac{c_{D}(z)}{\phi(z)} .
$$

In particular, $K_{D}(z, z)>0$.

Proof. We first reduce to the case when $\phi$ is continuous. The technique is standard. Let $D_{j}, j=1,2, \ldots$, be a sequence of bounded pseudoconvex domains so that $D_{j} \subset D_{j+1}, D=\bigcup_{j=1}^{\infty} D_{j}$. We choose a sequence of smooth functions $\phi_{j}$ defined on $D_{j}$ such that $-\log \phi_{j}$ is psh and $-\log \phi_{j} \downarrow-\log \phi$ as $j \rightarrow \infty$. Let $z \in D$ be an arbitrary point. Since

$$
\left\|K_{D_{j}, \phi_{j}}(\cdot, z) / K_{D_{j}, \phi_{j}}(z, z)^{1 / 2}\right\|_{D_{j}, \phi_{j}}=1
$$

there is a subsequence $K_{D_{j_{k}}, \phi_{j_{k}}}(\cdot, z) / K_{D_{j_{k}}, \phi_{j_{k}}}(z, z)^{1 / 2}$ which converges weakly to a holomorphic function $f$ on $D$ with $\|f\|_{D, \phi}=1$. For any holomorphic function $g$ on $D$ with $\|g\|_{D, \phi}=1$, we have

$$
\frac{|g(z)|}{\|g\|_{D_{j_{k}}, \phi_{j_{k}}}} \leq K_{D_{j_{k}}, \phi_{j_{k}}}(z, z)^{1 / 2} .
$$


Letting $k \rightarrow \infty$, we obtain $|g(z)| /\|g\|_{D, \phi} \leq f(z)$, which implies that $f(z)=$ $K_{D, \phi}(z, z)^{1 / 2}$. If we prove that

$$
K_{D_{j_{k}}, \phi_{j_{k}}}(z, z) \geq 2^{-8} \pi^{-1} \frac{c_{D}(z)}{\phi_{j_{k}}(z)},
$$

then the desired estimate is obtained by letting $k \rightarrow \infty$.

For any fixed $z \in D$ we set $D^{\prime}=\{z\}$ and $\Psi(\zeta)=2 n g_{D}(\zeta, z)-\log \phi(\zeta)$. Since $g_{D}(\zeta, z) \sim \log |\zeta-z|$ near $z$, we can take a real $\delta>0$ such that $\inf _{|\zeta-z|=\delta} g_{D}(\zeta, z)>-C_{\delta}$ where $C_{\delta}$ is a positive constant. Set

$$
u_{z}(\zeta)= \begin{cases}g_{D}(\zeta, z) & \text { if }|\zeta-z| \leq \delta \\ \max \left\{g_{D}(\zeta, z),-C_{\delta}\right\} & \text { if }|\zeta-z|>\delta\end{cases}
$$

We see that $g_{D}(\cdot, z) \in L_{\mathrm{loc}}^{\infty}(D \backslash\{z\})$ since $g_{D}(\zeta, z) \geq u_{z}(\zeta)$. Hence $\Psi \in \#\left(D^{\prime}\right)$ and furthermore,

$$
\frac{2 n}{\sigma_{2 n-1}} \int_{D} e^{-\Psi} \chi_{R(\Psi, t)} d V \leq\left(\frac{\phi(z)}{c_{D}^{1}(z)}+o(t)\right) \frac{2 n}{\sigma_{2 n-1}} \int_{M_{t}}|\zeta-z|^{-2 n} d V,
$$

since $\Psi^{-1}(-t-1,-t)$ is contained in

$$
\begin{array}{r}
M_{t}:=\left\{\zeta \in D:-t-1-\log c_{D}^{2}(z)+\log \phi(z)-o(t)<2 n \log |\zeta-z|\right. \\
\left.<-t-\log c_{D}^{1}(z)+\log \phi(z)+o(t)\right\} .
\end{array}
$$

Here we use $o(t)$ to denote any positive function which converges to zero as $t \rightarrow \infty$. Note that

$$
\frac{2 n}{\sigma_{2 n-1}} \int_{M_{t}}|\zeta-z|^{-2 n} d V=1+\log \frac{c_{D}^{2}(z)}{c_{D}^{1}(z)}+o(t)
$$

Hence

$$
\int_{D^{\prime}} d V[\Psi] \leq \frac{\phi(z)}{c_{D}(z)}
$$

Applying Proposition 3.1 to the constant function $f \equiv 1$ on $D^{\prime}$, we obtain a holomorphic function $F$ on $D$ so that $F(z)=1$ and

$$
\int_{D}|F|^{2} e^{-\Psi} d V \leq 2^{8} \pi \frac{\phi(z)}{c_{D}(z)}
$$

It follows that

$$
K_{\phi}(z, z) \geq \frac{|F(z)|^{2}}{\int_{D}|F|^{2} \phi d V} \geq \frac{1}{\int_{D}|F|^{2} e^{-\Psi} d V} \geq 2^{-8} \pi^{-1} \frac{c_{D}(z)}{\phi(z)}
$$

because $g_{D}(\zeta, z)<0$ on $D$. 
An immediate consequence is the following

Corollary 3.3. Let $D, \phi$ be as in Theorem 3.2 and $D_{m, \phi}$ be the Hartogs domain defined as in Section 2. Then

$$
K_{D_{m, \phi}}((z, t),(z, t)) \geq C_{m} \frac{\phi(z) c_{D}(z)}{\left(\phi(z)-|t|^{2}\right)^{m+1}}
$$

where $C_{m}>0$ is a constant depending only on $m$.

Proof. Ligocka's formula and Theorem 3.2 show

$K_{D_{m, \phi}}((z, t),(z, t)) \geq 2^{-8} \pi^{-1-m} c_{D}(z)^{2 n} \sum_{j=0}^{\infty}(j+1)(j+2) \ldots(j+m) \frac{|t|^{2 j}}{\phi^{m+j}}$.

By a simple computation, we obtain the desired result.

We also have

Corollary 3.4. Let $D \subset \mathbb{C}^{n}$ be a regular domain in the sense of Sibony [27], that is, there is a negative $C^{2}$ psh exhaustion function $u$ satisfying $\partial \bar{\partial} u \geq \partial \bar{\partial}|z|^{2}$. Then there is a universal constant $A>0$ such that

$$
K_{D}(z, z) \geq A(-u(z))^{-n} .
$$

REMARK. Note that the domain is not necessarily bounded.

Proof. Let $\theta$ be a smooth nondecreasing function on $\mathbb{R}^{+}$such that $\theta(x)=$ $x$ for $x \leq 1 / 2$ and $\theta(x)=1$ for $x \geq 3 / 4$. Let $A_{1}$ be an upper bound for the second derivative of $\log \theta$. For any $z \in D$ we set $\lambda_{z}=1 /|u(z)|$ and $r_{z}=A_{1}|u(z)|$ and

$$
\Psi_{z}(\zeta)=\theta\left(|\zeta-z|^{2} / r_{z}\right) \exp \left(\lambda_{z} u(\zeta)\right), \quad \zeta \in D
$$

A simple computation shows that $\log \Psi_{z}$ is a psh function on $D$ (cf. [27]). Notice that $\frac{1}{2} \log \Psi_{z}$ is a negative psh function with a logarithmic pole at $z$. Furthermore,

$$
\lim _{\zeta \rightarrow z}\left(\frac{1}{2} \log \Psi_{z}(\zeta)-\log |\zeta-z|\right)=\left(e A_{1}|u(z)|\right)^{-1} .
$$

The assertion then follows by an argument similar to, but simpler than, the proof of Theorem 3.2.

\section{Asymptotic behavior of the weighted Bergman kernel function}

Proof of Theorem 1.1. We proceed in two steps:

a) Upper limit: The proof is standard. Let $z \in D$ and $f \in H^{2}\left(D, \phi^{k} \psi\right)$. By the submean value theorem and the Cauchy-Schwarz inequality we obtain 


$$
\begin{aligned}
|f(z)| & \leq \frac{1}{\operatorname{vol}(B(z, \delta))} \int_{B(z, \delta)}|f| d V \\
& \leq \frac{1}{\operatorname{vol}(B(z, \delta))}\left(\int_{B(z, \delta)}|f|^{2} \phi^{k} \psi d V\right)^{1 / 2}\left(\int_{B(z, \delta)} \phi^{-k} \psi^{-1} d V\right)^{1 / 2} \\
& \leq \frac{1}{\operatorname{vol}(B(z, \delta))}\left(\int_{B(z, \delta)}|f|^{2} \phi^{k} \psi d V\right)^{1 / 2} \sup _{B(z, \delta)} \phi^{-k / 2} \psi^{-1 / 2}
\end{aligned}
$$

where $\delta>0$ is so small that $B(z, \delta) \subset D$. This implies that

$$
K_{\phi^{k} \psi}(z, z)^{1 / k} \leq \frac{1}{\operatorname{vol}(B(z, \delta))^{1 / k}} \sup _{B(z, \delta)} \phi^{-1} \psi^{-1 / k} .
$$

The local boundedness of $1 / \psi$ yields

$$
\limsup _{k \rightarrow \infty} K_{\phi^{k} \psi}(z, z)^{1 / k} \leq \sup _{B(z, \delta)} \phi^{-1} .
$$

Letting $\delta \rightarrow 0$, we have

$$
\limsup _{k \rightarrow \infty} K_{\phi^{k} \psi}(z, z)^{1 / k} \leq 1 / \phi(z)
$$

since $-\log \phi$ is psh (which implies that $1 / \phi$ is upper semicontinuous).

b) Suppose that the inequality $-k_{0} \log \phi-\log \psi \geq \lambda$ holds for some number $k_{0}>0$ and some $\lambda \in \operatorname{PSH}(D)$. Since the weighted Bergman kernel function is decreasing with respect to the weight, according to Theorem 3.2 one has, for all $k \geq k_{0}$,

$$
K_{\phi^{k} \psi}(z, z) \geq K_{\phi^{k-k_{0} e^{-\lambda}}}(z, z) \geq 2^{-8} \pi^{-1} c_{D}(z) e^{\lambda(z)} \phi(z)^{k_{0}-k}
$$

because $-\log \phi, \lambda \in \operatorname{PSH}(D)$. We immediately obtain

$$
\liminf _{k \rightarrow \infty} K_{\phi^{k} \psi}(z, z)^{1 / k} \geq 1 / \phi(z)
$$

since $c_{D}(z)>0$ and $\lambda \in L_{\mathrm{loc}}^{\infty}(D)$. The proof is complete.

In the theory of the Berezin quantization, one always takes $-\log \phi$ to be the potential of some Kähler metric $d s^{2}=\sum_{j, k} g_{j k} d z_{j} d \bar{z}_{k}$ and $\psi=$ $\operatorname{det}\left(g_{j \bar{k}}\right)$. We are interested in two classical metrics: the Kähler-Einstein metric and the Bergman metric. The Kähler-Einstein metric has "constant curvature" in the sense that $\operatorname{Ric}_{j \bar{k}}=C g_{j \bar{k}}$ for suitable constant $C$, where $\operatorname{Ric}_{j \bar{k}}=-\partial^{2} \log \psi / \partial z_{j} \partial \bar{z}_{k}$ is the Ricci tensor of the metric. Mok-Yau [22] showed that there exists a complete Kähler-Einstein metric on any bounded pseudoconvex domain in $\mathbb{C}^{n}$ with constant curvature $-(n+1)$, and it is unique (up to rescaling). This implies $-(n+1) \log \phi-\log \psi$ is pluriharmonic on $D$. Hence, by Theorem 1.1, we have

Corollary 4.1. Let $D$ be a bounded pseudoconvex domain in $\mathbb{C}^{n}$ equipped with the Kähler-Einstein metric. Then (1) holds. 
However, when $D$ is equipped with the Bergman metric, we can only obtain a weaker result.

Corollary 4.2. Let $D$ be a pseudoconvex domain such that

$$
K_{D}(z, z) \geq C_{\alpha} \delta_{D}(z)^{-\alpha}
$$

for some positive constants $\alpha, C_{\alpha}$. Then (1) also holds. Here $\delta_{D}(z)$ denotes the boundary distance of $z$.

REMARK. If $D$ is a bounded pseudoconvex domain in $\mathbb{C}^{n}$ with a Lipschitz boundary, then $K_{D}(z, z) \geq C_{\varepsilon} \delta_{D}(z)^{-2+\varepsilon}$ for any $\varepsilon>0$ (cf. [24]). Hence the above corollary applies in particular to any bounded pseudoconvex domain with Lipschitz boundary.

Proof of Corollary 4.2. Let $z \in D$ be fixed and let $f \in H^{2}(D)$ with $\|f\|_{D} \leq 1$. Take a number $a>0$ so that the polydisc

$$
P\left(z, a \delta_{D}(z)\right):=\left\{\zeta \in \mathbb{C}^{n}: \max \left\{\left|\zeta_{1}-z_{1}\right|, \ldots,\left|\zeta_{n}-z_{n}\right|\right\}<a \delta_{D}(z)\right\} \subset \subset D .
$$

By Cauchy's integral formula, we have

$$
\begin{aligned}
\frac{\partial f}{\partial z_{j}}(z)= & \frac{1}{(2 \pi i)^{n}} \int_{\partial P(z, r)} f\left(\zeta_{1}, \ldots, \zeta_{n}\right)\left(\zeta_{1}-z_{1}\right)^{-1} \\
& \ldots\left(\zeta_{j}-z_{j}\right)^{-2} \ldots\left(\zeta_{n}-z_{n}\right)^{-1} d \zeta_{1} \ldots d \zeta_{n}
\end{aligned}
$$

for any $1 \leq j \leq n, 0<r \leq a \delta_{D}(z)$. Therefore,

$$
\left|\frac{\partial f}{\partial z_{j}}(z)\right| \leq \frac{1}{(2 \pi)^{n}} \int_{\partial P(z, r)}\left|f\left(\zeta_{1}, \ldots, \zeta_{n}\right)\right| r_{1}^{-1} \ldots r_{j}^{-2} \ldots r_{n}^{-1}\left|d \zeta_{1} \ldots d \zeta_{n}\right| .
$$

Integrating on both sides we obtain

$$
\begin{aligned}
\left|\frac{\partial f}{\partial z_{j}}(z)\right|_{\frac{a}{2} \delta_{D}(z)}^{a \delta_{D}(z)} \ldots \int_{\frac{a}{2} \delta_{D}(z)}^{a \delta_{D}(z)} r_{1}^{2} \ldots r_{j}^{3} \ldots r_{n}^{2} d r_{1} \ldots d r_{n} & =\int_{P\left(z, a \delta_{D}(z)\right) \backslash P\left(z, \frac{a}{2} \delta_{D}(z)\right)}|f| d V \\
& \leq\left(\int_{D}|f|^{2} d V\right)^{1 / 2} \operatorname{vol}\left(P\left(z, a \delta_{D}(z)\right)\right)^{1 / 2} \leq \frac{C_{1}}{\delta_{D}(z)^{n}}
\end{aligned}
$$

where $C_{1}>0$ depends only on $a, n$. Let us recall a well known formula for the Bergman metric:

$d s^{2}(z, X)=K_{D}(z, z)^{-1} \sup \left\{|X f(z)|^{2}: f \in H^{2}(D), f(z)=0\right.$ and $\left.\|f\|_{D} \leq 1\right\}$ for any $z \in D, X \in \mathbb{C}^{n}$. We immediately establish

$$
\psi(z)=\operatorname{det}\left(g_{j \bar{k}}\right) \leq C_{2} K_{D}(z, z)^{-n} \delta_{D}(z)^{-2 n(4 n+1)}
$$


where $C_{2}>0$ depends also on $a, n$ only. Hence

$$
\begin{aligned}
-\log \psi & \geq-\log C_{2}+n \log K_{D}+2 n(4 n+1) \log \delta_{D} \\
& \geq-\log C_{2}+\frac{2 n(4 n+1)}{\alpha} \log C_{\alpha}-n\left(\frac{2(4 n+1)}{\alpha}-1\right) \log K_{D}
\end{aligned}
$$

because $K_{D}(z) \geq C_{\alpha} \delta_{D}(z)^{-\alpha}$. Let $k_{0}=n(2(4 n+1) / \alpha-1)$. Since $\phi=1 / K_{D}$, the function $-k_{0} \log \phi-\log \psi$ is bounded from below by a constant, hence Theorem 1.1 applies.

5. Zeroes of the Bergman kernel. Following an argument similar to the proof of Theorem 1.1, but simpler, we obtain

Lemma 5.1. Let $D$ be as in Theorem 1.1 and let $\left\{\phi_{k}\right\}_{k>0}$ be a sequence of positive functions on $D$ such that $-\log \phi_{k}$ are psh, $1 / \phi_{k} \in L_{\mathrm{loc}}^{\infty}(D)$ and $\phi_{k}^{1 / k}$ converge pointwise to a lower semicontinuous function $\phi$ on $D$. Then

$$
\lim _{k \rightarrow \infty} K_{\phi_{k}}(z, z)^{1 / k}=1 / \phi(z) .
$$

The following is a slight improvement of a theorem of Engliš [11]:

Lemma 5.2. Let $D, \phi_{k}, \phi$ be as above. Suppose that $1 / \phi$ cannot be extended as a sesqui-holomorphic function near some point $z^{0} \in D$. Then for any neighborhood $U$ of $z^{0}, K_{\phi_{k}}(z, w)$ has a zero in $U \times U$ for all sufficiently large $k$.

Proof. Suppose that $K_{\phi_{k}}(z, w)$ are zero-free in $U \times U$ for all $k$. We may assume that $U$ is simply connected. Then for every $k$, there is a sesquiholomorphic branch of $K_{\phi_{k}}(z, w)^{1 / k}$ on $U \times U$. Since $1 / \phi$ is locally bounded, by (2), $K_{\phi_{k}}(z, z)^{1 / k}$ is uniformly bounded on $U \times U$, therefore so is $K_{\phi_{k}}(z, w)^{1 / k}$ because

$$
\left|K_{\phi_{k}}(z, w)\right|^{2} \leq K_{\phi_{k}}(z, z) K_{\phi_{k}}(w, w) .
$$

Hence there would be a subsequence $K_{\phi_{k_{j}}}(z, w)^{1 / k_{j}}$ which converges uniformly on compact subsets of $U \times U$ to a sesqui-holomorphic function $L(z, w)$. Then $L(z, z)=1 / \phi(z)$, which is a contradiction.

Proof of Theorem 1.2. By Ligocka's formula, one has

$$
K_{\Omega_{m, \varphi}}((z, 0),(w, 0))=\frac{m !}{\pi^{m}} K_{\varphi^{m}}(z, w) .
$$

Let $\varphi=\phi$. Then the assertion of (i) follows immediately from Theorem 1.1 and Lemma 5.2. Similarly for $\varphi=\phi_{k}$, the assertion of (ii) follows from Lemmas 5.1 and 5.2.

EXAMPLE 1 . We take the unit disc $\Delta$ as the base domain and $\phi(z)=$ $1-|z|^{k}$ where $k \geq 1$ is not an even integer. It is easy to see that $-\log \phi$ is 
psh on $\Delta$ and $1 / \phi$ is not real-analytic at $z=0$ (which implies that it cannot be extended to a sesqui-holomorphic function). It follows from Theorem 1.2 that there is a positive integer $m(k)$ such that the Bergman kernel of the convex domain

$$
\Omega_{k}:=\left\{(z, t) \in \mathbb{C}^{m(k)+1}:|z|^{k}+\left|t_{1}\right|^{2}+\ldots+\left|t_{m(k)}\right|^{2}<1\right\}
$$

has a zero. Moreover, the domain $\Omega_{k}$ has a $C^{2}$ boundary and it is very "close" to the unit ball in $\mathbb{C}^{m(k)+1}$ as $k \rightarrow 2+$; however, $K_{\Omega_{k}}$ always has a zero which is also very "close" to the origin. Notice that for any sufficiently small $C^{\infty}$ pertubation of the unit ball in $\mathbb{C}^{n}$ (the dimension is fixed!), the Bergman kernel of such a domain is zero-free (cf. [15]). It is natural to ask whether there exists a Reinhardt domain of type $\left|z_{1}\right|^{2 \alpha_{1}}+\ldots+\left|z_{n}\right|^{2 \alpha_{n}}<1$, where all $\alpha_{j}$ are positive integers, such that the Bergman kernel has a zero.

EXAMPLE 2. Let

$$
\phi_{k}=\frac{1-\left|z_{1}\right|^{2}}{1+\left(1+\left|z_{1}\right|\right)^{2 k}+\left(1-\left|z_{1}\right|\right)^{2 k}}, \quad k=1,2, \ldots
$$

Define

$$
\Omega_{k}=\left\{\left(z_{1}, z_{2}\right) \in \mathbb{C}^{2}:\left|z_{2}\right|^{2}<\phi_{k}\left(z_{1}\right), z_{1} \in \Delta\right\} .
$$

We claim that the function

$$
-\log \phi_{k}=-\frac{1}{2} \log \left(1-\left|z_{1}\right|^{2}\right)+\frac{1}{2} \log \left(\left(1+\left|z_{1}\right|\right)^{2 k}+\left(1-\left|z_{1}\right|\right)^{2 k}\right)
$$

is subharmonic on $\Delta$. In fact, if we write

$$
\left(1+\left|z_{1}\right|\right)^{2 k}+\left(1-\left|z_{1}\right|\right)^{2 k}=2+\sum_{j=1}^{2 k} a_{2 j}\left|z_{1}\right|^{2 j}=: 2+\varphi_{k}\left(z_{1}\right)
$$

where $a_{2 j}>0$, then from the Cauchy-Schwarz inequality we obtain $\varphi_{k} \partial \bar{\partial} \varphi_{k}$ $-\partial \varphi_{k} \bar{\partial} \varphi_{k} \geq 0$. It follows that $\log \left(2+\varphi_{k}\right)$ is subharmonic, and hence so is $-\log \phi_{k}$. Clearly $\phi_{k}^{1 / k} \rightarrow\left(1+\left|z_{1}\right|\right)^{-1}$ as $k \rightarrow \infty$. Since $1+\left|z_{1}\right|$ is not real-analytic at $0, K_{\Omega_{k}}(z, w)$ has a zero for all sufficiently large $k$. Notice that the defining function of $\Omega_{k}$ can be written as

$$
\left|z_{2}\right|^{2}\left(\left(1+\left|z_{1}\right|\right)^{2 k}+\left(1-\left|z_{1}\right|\right)^{2 k}\right)+\left|z_{1}\right|^{2}+\left|z_{2}\right|^{2}<1 .
$$

Thus we obtain a bounded, smooth, algebraic, strictly pseudoconvex, complete Reinhardt domain in $\mathbb{C}^{2}$ for which the Lu Qi-Keng conjecture fails.

Problem. Is the Lu Qi-Keng conjecture valid for convex domains in $\mathbb{C}^{2}$ ?

By the classical scaling method, we obtain 
THEOREM 5.3. For every positive integer $k>0$, there are a positive integer $m(k)$ and a bounded convex domain $\Omega$ in $\mathbb{C}^{m(k)}$ with a $C^{k}$ boundary such that the zero set $\left\{(z, w) \in \Omega \times \Omega: K_{\Omega}(z, w)=0\right\}$ accumulates at $a$ point $\left(z^{0}, z^{0}\right)$, where $z^{0} \in \partial \Omega$.

Proof. Example 1 shows that for each positive integer $k$ there is another positive integer $m(k)$ such that the Bergman kernel of the domain

$$
\Omega_{k}:=\left\{(z, t) \in \mathbb{C}^{m(k)+1}:|z|^{k+1 / 2}+\left|t_{1}\right|^{2}+\ldots+\left|t_{m(k)}\right|^{2}<1\right\}
$$

has a zero $\left(\left(z^{*}, t^{*}\right),\left(w^{*}, s^{*}\right)\right) \in \Omega_{k} \times \Omega_{k}$. Clearly, $\Omega_{k}$ is a convex domain with $C^{k}$ boundary. Similarly to [7], we consider the domain

$$
U=\left\{\zeta \in \mathbb{C}^{m(k)+1}: \operatorname{Re} \zeta_{m(k)}+\left|\zeta_{0}\right|^{k+1 / 2}+\left|\zeta_{1}\right|^{2}+\ldots+\left|\zeta_{m(k)-1}\right|^{2}<0\right\} .
$$

Notice that $U$ is mapped by

$$
\Phi(\zeta)=\left(\frac{4^{1 / k+1 / 2} \zeta_{0}}{\left(\zeta_{m(k)}-1\right)^{2 / k+1 / 2}}, \frac{2 \zeta_{1}}{\zeta_{m(k)}-1}, \ldots, \frac{2 \zeta_{m(k)-1}}{\zeta_{m(k)}-1}, \frac{\zeta_{m(k)}+1}{\zeta_{m(k)}-1}\right)
$$

biholomorphically onto the domain $\Omega_{k}$, and the scaling map

$$
S_{\varepsilon}(\zeta)=\left(\varepsilon^{1 / k+1 / 2} \zeta_{0}, \sqrt{\varepsilon} \zeta_{1}, \ldots, \sqrt{\varepsilon} \zeta_{m(k)-1}, \varepsilon \zeta_{m(k)}\right)
$$

is a holomorphic automorphism of $U$ for arbitrary $\varepsilon>0$. Set $F_{\varepsilon}=\Phi \circ$ $S_{\varepsilon} \circ \Phi^{-1}$. Then $K_{\Omega_{k}}$ vanishes at all the points $\left(F_{\varepsilon}\left(z^{*}, t^{*}\right), F_{\varepsilon}\left(w^{*}, s^{*}\right)\right)$. Since $F_{\varepsilon}\left(z^{*}, t^{*}\right), F_{\varepsilon}\left(w^{*}, s^{*}\right) \rightarrow z^{0}=(0, \ldots, 0,-1) \in \partial \Omega_{k}$, the proof is complete.

REMARK. Since $K_{\Omega_{k}}(z, z) \rightarrow \infty$ as $z \rightarrow \partial \Omega_{k}$, the function $K_{\Omega_{k}}$ : $\overline{\Omega_{k} \times \Omega_{k}} \rightarrow \overline{\mathbb{C}}$ cannot be continuous.

6. Hilbert-Steinness of certain bounded domains. In 1970, Fischer [14] introduced the concept of Hilbert-Stein complex space in an attempt to prove an old conjecture of Serre (1953) that the holomorphic fiber bundle with a Stein base and a Stein fiber is Stein. He called a Stein space $F$ Hilbert-Stein if there exists a Hilbert space $H$ of holomorphic functions on $F$ which are invariant under the automorphism group Aut $F$ of $F$ and satisfy the following conditions: (i) $H$ separates points of $F$; (ii) $F$ is $H$-convex; (iii) for any map from a complex space $Y$ to Aut $F$ such that the associated map $Y \times F \rightarrow F$ is holomorphic, the induced map $Y \rightarrow$ Aut $H$ is holomorphic. It was shown in [14] that condition (iii) is trivially satisfied for all bounded domains in $\mathbb{C}^{n}$, and a bounded domain $D$ is Hilbert-Stein if every automorphism has bounded Jacobian determinant and $\lim _{z \rightarrow \partial D} K_{D}(z, z)=$ $\infty$. By Fefferman's well-known theorem [13], any bounded smooth strongly pseudoconvex domain is Hilbert-Stein. We prove 
TheOREM 6.1. Let $D$ be a bounded domain in $\mathbb{C}^{n}$. Suppose that there exists a point $w_{0} \in D$ such that:

(1) For any $h \in$ Aut $D$, there exists a positive constant $C^{\prime}$ depending only on $w_{0}$ and $h$ such that

$$
\left|K_{D}\left(z, h^{-1}\left(w_{0}\right)\right)\right| \leq C^{\prime}\left|K_{D}\left(z, w_{0}\right)\right|
$$

(2) There exists a point $w_{0} \in D$ such that $\lim _{z \rightarrow \partial D} K_{w_{0}}(z, z)=\infty$, where $K_{w_{0}}(z, z)$ denotes the Bergman kernel function associated to the weight function $\left|K_{D}\left(z, w_{0}\right)\right|^{2}$.

Then $D$ is Hilbert-Stein.

Proof. Since $D$ is bounded, $K_{D}\left(z, w_{0}\right)$ is not identically zero. Hence $\left|K_{D}\left(z, w_{0}\right)\right|^{-1 / 2}$ is locally integrable, which implies that $\left|K_{D}\left(z, w_{0}\right)\right|^{2}$ is an admissible weight. We set

$$
H=\left\{f \in \mathcal{O}(D): \int_{D}|f(z)|^{2}\left|K_{D}\left(z, w_{0}\right)\right|^{2} d V_{z}<\infty\right\} .
$$

Since $\int_{D}\left|K_{D}\left(z, w_{0}\right)\right|^{2} d V_{z}=K_{D}\left(w_{0}, w_{0}\right)$, we conclude that $H$ contains all bounded holomorphic functions. Hence (i) is satisfied because bounded holomorphic functions separate points of $D$. Let $h \in$ Aut $D$. For any $f \in H$, one has

$$
\begin{aligned}
\int_{D}|f(h(z))|^{2}\left|K_{D}\left(z, w_{0}\right)\right|^{2} d V_{z} & =\int_{D}|f(\zeta)|^{2}\left|K_{D}\left(\zeta, h^{-1}\left(w_{0}\right)\right)\right|^{2}\left|J_{h}\left(w_{0}\right)\right|^{-2} d V_{\zeta} \\
& \leq C^{\prime \prime} \int_{D}|f(\zeta)|^{2}\left|K_{D}\left(\zeta, w_{0}\right)\right|^{2} d V_{\zeta}<\infty
\end{aligned}
$$

where the last inequality follows from condition (1). This implies that $H$ is invariant under Aut $D$. It suffices to show that $D$ is $H$-convex. Suppose that there is a sequence $\left\{w_{k}\right\}_{k=1}^{\infty}$ of points in $D$ such that $\sup _{k}\left|f\left(w_{k}\right)\right|<\infty$ for any $f \in H$. It follows immediately that $\sup _{k} K_{w_{0}}\left(w_{k}, w_{k}\right)<\infty$. This contradicts condition (2). The proof is complete.

REMARK. Condition (2) holds in particular when $\lim _{z \rightarrow \partial D} K_{D}(z, z)=$ $\infty$ and $\left|K_{D}\left(z, w_{0}\right)\right|$ is bounded. This includes for example all bounded pseudoconvex domains of finite type and all bounded pseudoconvex complete Reinhardt domains (cf. [1]). However, condition (1) is rather difficult to check. Just as with Lu Qi-Keng domains, one can only obtain a few examples which satisfy the conditions of the theorem. Based on the explicit expression of the Bergman kernel, it is not difficult to verify these conditions for balls, polydiscs and the Tullen domain $\left\{z \in \mathbb{C}^{2}:\left|z_{1}\right|^{2}+\left|z_{2}\right|^{p}<1\right\}$, $p>0$. However, in these cases all automorphisms have bounded Jacobian determinants. Hence Theorem 6.1 gives no new results for these cases. 


\section{Proofs of Theorems 1.3 and 1.4}

1. Quantitative estimates of the pluricomplex Green function. Recently, it was proved that any bounded pseudoconvex domain is complete with respect to the Bergman metric if the volume of the sublevel set

$$
A(w, D):=\left\{z \in D: g_{D}(z, w)<-1\right\}
$$

tends to zero as $w \rightarrow \partial D$ (cf. [5], [16]). This implies the Bergman completeness of bounded hyperconvex domains [3], [16]. In this spirit, DiederichHerbort gave a quantitative estimate of this sublevel set for certain pseudoconvex domains.

Proposition 7.1 (see [8]). Let $D$ be as in Theorem 1.4. Then for any fixed number $0<t \ll 1$ there is a number $0<\delta_{t} \ll 1$ such that for all $w \in D$ with $\delta_{D}(w)<\delta_{t}$,

$$
A(w, D) \subset\left\{z \in D: \delta_{D}(w)^{1+t}<\delta_{D}(z)<\delta_{D}(w)^{1-t}\right\} .
$$

In fact, Diederich-Herbort only proved that

$$
A(w, D) \subset\left\{z \in D: \delta_{D}(z)<\delta_{D}(w)^{1-t}\right\} .
$$

However, the other part is implicit in their paper. By Lemma 2.4 in [9], there is a constant $\widetilde{C}=\widetilde{C}(D)$ such that for any compact set $K \subset D$,

$$
\sup _{w \in K}\left|g_{D}(z, w)\right| \leq \widetilde{C} \delta_{D}(K)^{-\alpha} \delta_{D}(z)^{\alpha} \log \frac{2 \operatorname{dim}(D)}{\delta_{D}(K)}
$$

for all $z \in D$ with $\delta_{D}(z) \leq \frac{1}{2} \delta_{D}(K)$. Here $\delta_{D}(K)$ denotes the distance between $K$ and $\partial D$, and $\alpha$ is as in Theorem 1.4. Let now $w \in D$ be arbitrary and take $K=\left\{z \in D: \delta_{D}(z) \geq \delta_{D}(w)\right\}$. One has

$$
\begin{aligned}
A(w, D) & \subset\left\{z \in D: \delta_{D}(z)>\frac{1}{2} \delta_{D}(w)\right\} \\
& \cup\left\{z \in D: \delta_{D}(z) \leq \frac{1}{2} \delta_{D}(w), \widetilde{C} \delta_{D}(w)^{-\alpha} \delta_{D}(z)^{\alpha} \log \frac{2 \operatorname{dim}(D)}{\delta_{D}(w)}>1\right\},
\end{aligned}
$$

from which the assertion follows immediately.

For convex domains, we can obtain a slightly stronger result.

Proposition 7.2. Let $D$ be a bounded convex domain in $\mathbb{C}^{n}$. Then

$$
A(w, D) \subset\left\{z \in D: C^{-1} \delta_{D}(w)<\delta_{D}(z)<C \delta_{D}(w)\right\} .
$$

Proof. Let $w \in D$. Take a boundary point $w^{*}$ so that $\left|w-w^{*}\right|=\delta_{D}(w)$. Since $D$ is convex, there exists a linear function $h_{w^{*}}$ such that $h_{w^{*}}\left(w^{*}\right)=0$ and $D \subset\left\{z \in \mathbb{C}^{n}: \operatorname{Re} h_{w^{*}}(z)<0\right\}$. Set $f_{w^{*}}=e^{h_{w^{*}}}$. Since $\left|h_{w^{*}}\right|<1$ on $D$, one has 


$$
\begin{aligned}
-g_{D}(z, w) & \leq-g_{\Delta}\left(f_{w^{*}}(z), f_{w^{*}}(w)\right) \leq-\log \frac{\left|f_{w^{*}}(z)-f_{w^{*}}(w)\right|}{\left|1-\overline{f_{w^{*}}(w)} f_{w^{*}}(z)\right|} \\
& \leq \frac{1}{2} \log \left(1+\frac{\left(1-\left|f_{w^{*}}(z)\right|^{2}\right)\left(1-\left|f_{w^{*}}(w)\right|^{2}\right)}{\left|f_{w^{*}}(z)-f_{w^{*}}(w)\right|^{2}}\right) \\
& \leq \frac{\left(1-\left|f_{w^{*}}(z)\right|^{2}\right)\left(1-\left|f_{w^{*}}(w)\right|^{2}\right)}{2\left|f_{w^{*}}(z)-f_{w^{*}}(w)\right|^{2}} \\
& \leq 2 \frac{\left(1-\left|f_{w^{*}}(z)\right|\right)\left(1-\left|f_{w^{*}}(w)\right|\right)}{\left|f_{w^{*}}(z)-f_{w^{*}}(w)\right|^{2}}
\end{aligned}
$$

where $\Delta$ is the unit disc. If $1-\left|f_{w^{*}}(z)\right| \geq 2\left(1-\left|f_{w^{*}}(w)\right|\right)$, then

$$
\left|f_{w^{*}}(z)-f_{w^{*}}(z)\right| \geq 1-\left|f_{w^{*}}(z)\right|-\left(1-\left|f_{w^{*}}(w)\right|\right) \geq \frac{1}{2}\left(1-\left|f_{w^{*}}(z)\right|\right) .
$$

This implies

$$
-g_{D}(z, w) \leq 8 \frac{1-\left|f_{w^{*}}(w)\right|}{1-\left|f_{w^{*}}(z)\right|}
$$

Hence

$$
A(w, D) \subset\left\{z \in D: 1-\left|f_{w^{*}}(z)\right| \leq 8\left(1-\left|f_{w^{*}}(w)\right|\right)\right\} .
$$

Notice that $1-\left|f_{w^{*}}(w)\right| \leq \lambda_{1} \delta_{D}(w)$ and $1-\left|f_{w^{*}}(z)\right| \geq \lambda_{2} \delta_{D}(z)$ because $D$ is convex. Here $\lambda_{1}, \lambda_{2}$ are two positive constants depending only on $D$. Hence

$$
A(w, D) \subset\left\{z \in D: \delta_{D}(z)<C \delta_{D}(w)\right\}
$$

where $C=8 \lambda_{1} / \lambda_{2}$. The conclusion follows from the symmetry of $g_{D}$ for convex domains.

2. Proofs of the theorems. We first prove Theorem 1.4. The main part of the proof is to show the following two statements:

Lemma 7.3. (i) For any $s \geq 0$, one has

$$
\frac{K_{s}(z, z)}{K_{D}(z, z)} \geq C^{-1} \inf _{A(z, D)} \delta_{D}^{-s} .
$$

(ii) For any $s>-\alpha$, one has

$$
\frac{K_{D}(z, z)}{K_{s}(z, z)} \geq C^{-1} \inf _{A(z, D)} \delta_{D}^{s}
$$

Proof. Set $\phi_{z}=-\log \left(-g_{D}(\cdot, z)+1\right)$ for fixed $z \in D$. Define

$$
\varphi_{z}= \begin{cases}-s \log \delta_{D}+2 n g_{D}(\cdot, z)+\phi_{z} & \text { for case (i), } \\ 2 n g_{D}(\cdot, z)+\phi_{z} & \text { for case (ii). }\end{cases}
$$

Clearly, $\varphi_{z}$ is psh on $D$. Let $\chi: \mathbb{R} \rightarrow[0,1]$ be a cut-off function such that $\chi=1$ on $(-\infty,-2)$ and $\chi=0$ on $(-1, \infty)$. Set

$$
\eta= \begin{cases}\chi\left(\phi_{z} / \log 2\right) K_{D}(\cdot, z) / K_{D}(z, z)^{1 / 2} & \text { for case (i) } \\ \chi\left(\phi_{z} / \log 2\right) K_{s}(\cdot, z) / K_{s}(z, z)^{1 / 2} & \text { for case (ii) }\end{cases}
$$


We will carry out the proof under the assumption that $\phi_{z}, \varphi_{z}$ are $C^{2}$; the general case follows from a standard limiting procedure. Observe that

$$
\left|\bar{\partial} \chi\left(\phi_{z} / \log 2\right)\right|_{\partial \bar{\partial} \varphi_{z}} \leq \sup \left|\chi^{\prime}\right| / \log 2
$$

because $\partial \bar{\partial} \varphi_{z} \geq \partial \bar{\partial} \phi_{z} \geq \partial \phi_{z} \bar{\partial} \phi_{z}$. We solve the equation $\bar{\partial} u=\bar{\partial} \eta$ as in Lemma 4.4.1 of [17] together with the estimate

$$
\begin{aligned}
& \int_{D}|u|^{2} e^{-\varphi_{z}} d V \leq \int_{D}|\bar{\partial} \eta|_{\partial \bar{\partial} \varphi_{z}}^{2} e^{-\varphi_{z}} d V \\
& \leq C \begin{cases}\int_{A(z, D)}\left|K_{D}(\cdot, z) / K_{D}(z, z)^{1 / 2}\right|^{2} \delta_{D}^{s} d V & \text { for case (i), } \\
\int_{A(z, D)}\left|K_{s}(\cdot, z) / K_{s}(z, z)^{1 / 2}\right|^{2} d V & \text { for case (ii), }\end{cases} \\
& \leq C \begin{cases}\sup _{A(z, D)} \delta_{D}^{s} & \text { for case (i), } \\
\sup _{A(z, D)} \delta_{D}^{-s} & \text { for case (ii). }\end{cases}
\end{aligned}
$$

Set $F=\eta-u$. Then $F$ is holomorphic on $D$ and

$$
F(z)= \begin{cases}K_{D}(z, z)^{1 / 2} & \text { for case (i) } \\ K_{s}(z, z)^{1 / 2} & \text { for case (ii) }\end{cases}
$$

because $g_{D}(\zeta, z) \sim \log |\zeta-z|$ near $z$. Furthermore, for case (i) one has

$$
\begin{aligned}
\int_{D}|F|^{2} \delta_{D}^{s} d V & \leq 2 \int_{D}|\eta|^{2} \delta_{D}^{s} d V+2 \int_{D}|u|^{2} \delta_{D}^{s} d V \\
& \leq 2 \int_{D}|\eta|^{2} \delta_{D}^{s} d V+2 \int_{D}|u|^{2} e^{-\varphi_{z}} d V \leq C \sup _{A(z, D)} \delta_{D}^{s}
\end{aligned}
$$

because $2 n g_{D}(\cdot, z)+\phi_{z}<0$ on $D$. Similarly for case (ii) one has

$$
\int_{D}|F|^{2} d V \leq C \sup _{A(z, D)} \delta_{D}^{-s} .
$$

The assertion of the lemma follows immediately from the following fact:

$$
\begin{array}{ll}
K_{s}(z, z) \geq \frac{|F(z)|^{2}}{\int_{D}|F|^{2} \delta_{D}^{s} d V} & \text { for case (i), } \\
K_{D}(z, z) \geq \frac{|F(z)|^{2}}{\int_{D}|F|^{2} d V} & \text { for case (ii). }
\end{array}
$$

Lemma 7.4. For any $-\alpha<s<0$, one has

$$
\frac{K_{s}(z, z)}{K_{D}(z, z)} \geq C_{s}^{-1} \inf _{A(z, D)} \delta_{D}^{-s} .
$$


Proof. Set $\psi=(s / \alpha) \log (-\varrho)$. It is a psh function which satisfies

$$
\frac{-s}{\alpha} \partial \bar{\partial} \psi \geq \partial \psi \bar{\partial} \psi \text {. }
$$

Let $\phi_{z}, \chi$ be as above. We set

$$
\varphi_{z}=2 n g_{D}(\cdot, z)+\phi_{z}, \quad \eta=\chi\left(\phi_{z} / \log 2\right) K_{D}(\cdot, z) / K_{D}(z, z)^{1 / 2} .
$$

We will use a generalized Donnelly-Fefferman estimate (cf. [2]):

Proposition 7.5. Let $D$ be a bounded pseudoconvex domain in $\mathbb{C}^{n}$, let $\varphi, \psi \in \operatorname{PSH}(D)$ and assume that

$$
r \partial \bar{\partial} \psi \geq \partial \psi \bar{\partial} \psi
$$

in the distribution sense for suitable $0<r<1$ (in other words, the function $-e^{-\psi / r}$ is $\left.p s h\right)$. Then for any $\bar{\partial}$-closed $(0,1)$-form $g$ in $D$ there is a solution $u$ to the equation $\bar{\partial} u=g$ such that

$$
\int_{D}|u|^{2} e^{-\varphi+\psi} d V \leq C_{r} \int_{D}|g|_{\partial \bar{\partial}(\varphi+\psi)}^{2} e^{-\varphi+\psi} d V .
$$

Applying this proposition with $\varphi=\varphi_{z}, r=-s / \alpha$, we obtain a solution to the equation $\bar{\partial} u=\bar{\partial} \eta$ together with the estimate

$$
\begin{aligned}
\int_{D}|u|^{2} e^{\psi-\varphi} d V & \leq C_{s} \int_{D}|\bar{\partial} \eta|_{\partial \bar{\partial}(\varphi+\psi)}^{2} e^{\psi-\varphi} d V \\
& \leq C_{s} \int_{A(z, D)}\left|K_{D}(\cdot, z) / K_{D}(z, z)^{1 / 2}\right|^{2} \delta_{D}^{s} d V \\
& \leq C_{s} \sup _{A(z, D)} \delta_{D}^{s}
\end{aligned}
$$

because $e \psi \approx \delta_{D}^{s}$. We set $F=\eta-u$. A similar argument to the above completes the proof of the lemma.

Proof of Theorem 1.4. The statement follows directly from Proposition 7.1 and Lemmas 7.3 and 7.4.

Proof of Theorem 1.3. The localization property of the weighted Bergman kernel function reduces the proof to the case when $D$ is convex. Let $P_{D}$ be the Minkowski functional of $D$. Then $\varrho:=P_{D}-1$ is a negative psh exhaustion function satisfying

$$
C^{-1} \delta_{D}(z) \leq-\varrho(z) \leq C \delta_{D}(z) .
$$

Then the assertions of Lemmas 7.3 and 7.4 still hold with $\alpha$ replaced by 1 . The proof is complete by Proposition 7.2.

Acknowledgements. The author would like to thank the referee for valuable suggestions. 


\section{References}

[1] S. R. Bell and H. P. Boas, Regularity of the Bergman projection in weakly pseudoconvex domains, Math. Ann. 257 (1981), 23-30.

[2] B. Berndtsson and P. Charpentier, A Sobolev mapping property of the Bergman kernel, Math. Z. 235 (2000), 1-10.

[3] Z. Błocki and P. Pflug, Hyperconvexity and Bergman completeness, Nagoya J. Math. 151 (1998), 221-225.

[4] H. P. Boas, Lu Qi-Keng's problem, J. Korean Math. Soc. 37 (2000), 253-267.

[5] B. Y. Chen, Completeness of the Bergman metric on non-smooth pseudoconvex domains, Ann. Polon. Math. 71 (1999), 241-251.

[6] K. Diederich and J. E. Fornæss, Pseudoconvex domains: Bounded strictly plurisubharmonic exhaustion functions, Invent. Math. 39 (1977), 129-141.

[7] K. Diederich and G. Herbort, On discontinuity of the Bergman kernel function, Internat. J. Math. 10 (1999), 825-832.

[8] -, Quantitative estimates of the Green function and an application to the Bergman metric, Ann. Inst. Fourier (Grenoble) 50 (2000), 1205-1228.

[9] K. Diederich, G. Herbort and V. Michel, Weights of holomorphic extension and restriction, J. Math. Pures Appl. 77 (1998), 697-719.

[10] S. Dragomir, On weighted Bergman kernels of bounded domains, Studia Math. 108 (1994), 149-157.

[11] M. Engliš, Asymptotic behavior of reproducing kernels of weighted Bergman spaces, Trans. Amer. Math. Soc. 349 (1997), 3717-3735.

[12] - Zeroes of the Bergman kernel of Hartogs domains, Comment. Math. Univ. Carolin. 41 (2000), 199-202.

[13] C. Fefferman, The Bergman kernel and biholomorphic equivalence of pseudoconvex domains, Invent. Math. 26 (1974), 1-65.

[14] G. Fischer, Hilbert spaces of holomorphic functions on bounded domains, Manuscripta Math. 3 (1970), 305-314.

[15] R. E. Greene and S. G. Krantz, Stability properties of the Bergman kernel and curvature properties of bounded domains, in: Recent Developments in Several Complex Variables, Princeton Univ. Press, 1981, 179-198.

[16] G. Herbort, The Bergman metric on hyperconvex domains, Math. Z. 232 (1999), 183-196.

[17] L. Hörmander, An Introduction to Complex Analysis in Several Variables, NorthHolland, 1990.

[18] M. Klimek, Extremal plruisubharmonic functions and invariant pseudodistances, Bull. Soc. Math. France 113 (1985), 123-142.

[19] S. Kobayashi, Geometry of bounded domains, Trans. Amer. Math. Soc. 92 (1959), 267-290.

[20] E. Ligocka, On the Forelli-Rudin construction and weighted Bergman projections, Studia Math. 94 (1989), 257-272.

[21] Q. K. Lu, On Kähler manifolds with constant curvature, Acta Math. Sinica 16 (1966), 269-281.

[22] N. Mok and S. T. Yau, Completeness of the Kähler-Einstein metric on bounded domains and characterization of domains of holomorphy by curvature conditions, in: Proc. Sympos. Pure Math. 39, Amer. Math. Soc., 1983, 41-60.

[23] T. Ohsawa, Boundary behavior of the Bergman kernel function on pseudoconvex domains, Publ. RIMS Kyoto Univ. 20 (1984), 897-902. 
[24] T. Ohsawa, On the extension of $L^{2}$ holomorphic functions $V$. Effects of generalization, Nagoya Math. J. 161 (2001), 1-21.

[25] Z. Pasternak-Winiarski, On weights which admit the reproducing kernel of Bergman type, Internat. J. Math. Math. Sci. 15 (1992), 1-14.

[26] P. Pflug, Quadratintegrable holomorphe Funktionen und die Serre-Vermutung, Math. Ann. 216 (1975), 285-288.

[27] N. Sibony, A class of hyperbolic manifolds, in: Recent Developments in Several Complex Variables, Princeton Univ. Press, 1981, 357-372.

[28] —, Une classe de domaines pseudoconvexes, Duke Math. J. 55 (1987), 299-319.

Department of Applied Mathematics

Tongji University

200092 Shanghai, P.R. China

E-mail: chenbo-yong@lycos.com

Received November 12, 2001

Revised version June 3, 2002 